\title{
Signature of edge states in resonant wave scattering
}

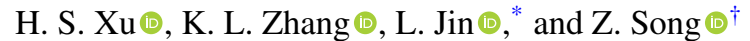 \\ School of Physics, Nankai University, Tianjin 300071, China
}

(Received 6 September 2021; revised 27 January 2022; accepted 18 February 2022; published 1 March 2022)

\begin{abstract}
Particle beam scattering is a conventional technique for detecting the nature of matter. We studied the scattering problem of a cluster connected to multiple leads. We established the connection between the eigenstate of the topological scattering center and the transmission and reflection amplitudes for the resonant scattering process. We discovered that, as an application, this approach enables the detection of the edge state in the band gap for both Hermitian and non-Hermitian systems and the identification of the topology of a system. We investigated two types of Su-Schrieffer-Heeger chains as examples. In addition, we proposed a dynamic scheme through an evanescently coupled-waveguide array to detect the edge state on the basis of measured transmission intensity. Numerical simulation revealed that pattern visibility can be the signature of the edge states.
\end{abstract}

DOI: 10.1103/PhysRevA.105.033501

\section{INTRODUCTION}

Studies on the integer quantum Hall effect have indicated that edge modes exist in the topological phase with nonzero Chern numbers [1]. According to band theory, the gap closing between filled and empty bands will motivate the existence of edge states [2]. The result is a robust feature for any form of boundary, called the bulk-boundary correspondence [3]. The only restriction is that, in cases where the topological invariant relies on an underlying symmetry, this symmetry must be preserved also in the boundary region. A typical example is the Su-Schrieffer-Heeger (SSH) model, in which chiral symmetry is crucial [4]. Another key feature of the edge state is that its wave function exponentially localizes on the boundary. The almost-zero-energy eigenstates of the SSH model are odd and even superpositions of states localized exponentially on the left and right edges. This is a consequence of the exponentially small overlap between the left and right edge states.

Photonic crystal is an excellent platform for the study of topological physics [5]. Topological photonic devices have been realized in microwave-scale magnetic photonic crystals [6-9] and meta-atom structures [10], functioning at optical and infrared frequencies in waveguide lattices [11] and resonator lattices $[12,13]$. Theoretical proposals based on modulated photonic crystal resonances [14], circuit quantum electrodynamics systems $[15,16]$, and metamaterial photonic crystals [17] have also been developed. The key feature of these devices is the existence of topologically protected electromagnetic edge states.

\footnotetext{
*jinliang@nankai.edu.cn

${ }^{\dagger}$ songtc@nankai.edu.cn
}

Published by the American Physical Society under the terms of the Creative Commons Attribution 4.0 International license. Further distribution of this work must maintain attribution to the author(s) and the published article's title, journal citation, and DOI.
A topological phase transition (TPT), which is caused by changes in the topology of the bulk band structure, differs considerably from common phase transitions such as the melting of a solid, which are characterized by broken symmetries and sharp anomalies in thermodynamic properties [3]. Numerous theoretical studies have demonstrated that TPTs can be induced by tuning the band structure through chemical substitution, strain, or pressure; via electron correlation effects [18-23]; and via laser or microwave pumping to produce a nonequilibrium topological state or Floquet topological insulator [24-32].

Topological states are characterized by topological invariants [33]. In fermionic systems, conductance measurements reveal these integer invariants. However, direct measurement of these integers is nontrivial in bosonic systems, mainly because the concept of conductance is not well defined $[34,35]$. Whereas these integers have been measured in one-dimensional (1D) bosonic systems [36-38], the twodimensional (2D) bosonic case has been realized in atomic lattices [39] and photonic systems [40].

Recently, topological concepts have been applied to scattering. Topological Fano resonance is immune to impurities, although it remains sensitive to system parameters [41]. A receiver protector was proposed and demonstrated employing a topological interface state of the SSH lattice [42]. Scattering methods to measure topological invariants have also been developed. The winding number of scattering matrix eigenvalues determines the number of edge states and topological invariants [43-48]. The relationships among surface scattering properties, bulk band properties, and the formation of interface states for a 1D centrosymmetric photonic crystal have been revealed [49]. In addition, the phase of the reflection coefficient can be used to measure the topological indices of a photonic system [50,51].

In this paper, we investigated the relationship between the transmission coefficients of multiple output channels scattering in the resonance process and the edge state of the topological scattering center, which can be used to intuitively 


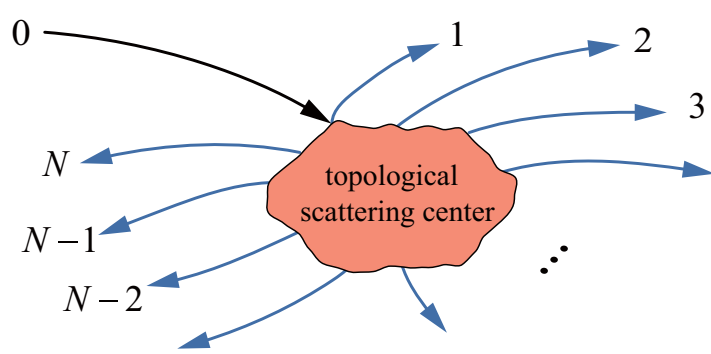

FIG. 1. Illustration of the multitransmission channel scattering system for detecting in-gap eigenstates. The orange area indicates $N$-site topological scattering center $H_{\mathrm{c}}$, and each site $|l\rangle_{\mathrm{c}}$ in the scattering center is connected to $l$ th transmission chain $(l \in[1, N])$. The incident and transmission chains are both tight-binding chains, and coupling strength $|J| \ll 1$.

detect the edge state. The proposed method is insensitive to system perturbation. We also developed a method of measuring real eigenenergy by determining whether perfect transmission occurs in a two-lead scattering system. We demonstrated the effectiveness of this method by applying it to detect an edge state in an SSH model and designed a corresponding experimental platform through a system of waveguide arrays. Through numerical simulation, we show that the proposed scattering system can be used to distinguish different phase regions from the visibility and reflection. We also show that our proposed scattering formalism can be used to detect the eigenfunctions of non-Hermitian systems with real energy spectra.

The remainder of this paper is organized as follows. In Sec. II, we design a multitransmission channel scattering system and find the relationship between transmission coefficients and the eigenfunction of the topological scattering center. In Sec. III, we take an SSH chain as scattering center and prove that our conclusion is applicable to detect the edge state. In Sec. IV, we provide the scheme to experimentally detect the edge state via a system of waveguide arrays, and investigate the visibility and reflection to identify different phase regions by numerical simulations. In Sec. V, we consider a non-Hermitian SSH chain by adding the imaginary potential $\pm i \gamma$, and we show the transmission probability in each lead is conformed to its eigenfunction, which is a sinusoidal function. Finally, we summarize the results and conclude in Sec. VI.

\section{GENERAL FORMALISM}

In this section, we provide the general scattering formalism for the detection of in-gap eigenstates. The transmission coefficients for the on-resonance input when all output channels are weakly connected to the scattering center approximately indicate the eigenstates of the scattering center. Figure 1 presents the structure of the proposed scattering system. The Hamiltonian of the system reads

$$
H=H_{\mathrm{c}}+H_{\text {in }}+H_{\text {out }}+H_{\text {jnt }},
$$

where $H_{\mathrm{c}}$ represents the topological scattering center, namely, an $N$-site lattice, and each site is attached to one output channel. The channels are semi-infinite tight-binding chains with uniform coupling strength $J$. We only considered cases with single input channels. The Hamiltonian of the input channel is

$$
H_{\text {in }}=\sum_{j=1}^{\infty}\left(J|-j\rangle_{0}\left\langle-j-\left.1\right|_{0}+\text { H.c. }+\mu \mid-j\right\rangle_{0}\left\langle-\left.j\right|_{0}\right),\right.
$$

where $|j\rangle_{0}$ is the single-particle basis of the input leads at site j. $H_{\text {out }}$ represents transmission channels and reads

$$
H_{\mathrm{out}}=\sum_{l=1}^{N} \sum_{j=1}^{\infty}\left(J|j\rangle_{l}\left\langle j+\left.1\right|_{l}+\text { H.c. }+\mu \mid j\right\rangle_{l}\left\langle\left. j\right|_{l}\right),\right.
$$

where $|j\rangle_{l}$ is the basis at site $j$ in the $l$ th output lead. The joint Hamiltonian is

$$
H_{\text {jnt }}=J|-1\rangle_{0}\left\langle\left. 1\right|_{\mathrm{c}}+J \sum_{l=1}^{N} \mid 1\right\rangle_{l}\left\langle\left. l\right|_{\mathrm{c}}+\text { H.c. },\right.
$$

where $|l\rangle_{\mathrm{c}}$ denotes the basis of scattering center $H_{\mathrm{c}}$ connected to the $l$ th transmission channel. Here, $J$ denotes the hopping strength and $\mu$ denotes the chemical potential for each lead. The entire scattering system consists of the scattering center and the input and output leads. Energy exchange between the leads and the scattering center is enabled by weak couplings.

The wave function for the input lead is represented by $\psi_{0}^{k}$, and the $l$ th output lead is represented by $\psi_{l}^{k}$, where $k$ is the dimensionless wave vector. The incoming plane wave is reflected and transmitted by the scattering center, and only outgoing plane waves are present in all output leads. The wave functions in the leads are given by

$$
\begin{aligned}
& \psi_{0}^{k}(j)=e^{i k j}+r e^{-i k j}, j<0 \\
& \psi_{l}^{k}(j)=t_{l} e^{i k j}, j>0,
\end{aligned}
$$

where $r$ and $t_{l}$ are the reflection and transmission coefficients for the input and $l$ th output leads, respectively. The corresponding wave function in the scattering center is represented by $\psi_{\mathrm{c}}^{k}(j)(j \in[1, N])$. According to the continuity condition of the wave function of a discrete quantum system, $\psi_{\mathrm{c}}^{k}(1)=$ $1+r$ and $\psi_{\mathrm{c}}^{k}(l)=t_{l}(l \in[1, N])$ because of Eq. (5). Thus,

$$
1+r=t_{1} \text {. }
$$

The leads are tight-binding chains with a uniform hopping strength and identical chemical potential. Dispersion relation $E_{k}=2 J \cos k+\mu$ can be obtained from the Schrödinger equations for lead Hamiltonians $H_{\text {in }}$ and $H_{\text {out }}$.

Accordingly, the Schrödinger equation for scattering center $H_{\mathrm{c}}$ is

$$
\left(H_{\mathrm{c}}-E_{k}\right)\left(\begin{array}{c}
t_{1} \\
t_{2} \\
\vdots \\
t_{N-1} \\
t_{N}
\end{array}\right)=-J\left(\begin{array}{c}
\left(e^{-i k}+r e^{i k}\right)+t_{1} e^{i k} \\
t_{2} e^{i k} \\
\vdots \\
t_{N-1} e^{i k} \\
t_{N} e^{i k}
\end{array}\right) \text {, }
$$

which connects the eigenproblem of $H_{\mathrm{c}}$ to the scattering problem. For a given explicit form $H_{\mathrm{c}}, r$ and $t_{l}$ can be obtained. This analysis is not restricted to a Hermitian $H_{\mathrm{c}}$. We focused on obtaining a solution with $k \in[0, \pi]$ and real $\mu$, which requires that the corresponding eigenenergy be real. 
Equation (7) reduces to the secular equation of $H_{\mathrm{c}}$,

$$
\left(H_{\mathrm{c}}-E_{k}\right)\left(t_{1}, \quad t_{2}, \quad \cdots, \quad t_{N-1}, \quad t_{N}\right)^{T}=0,
$$

in the limit of zero $J$. When the input plane wave is in resonance with the eigenenergy of $H_{\mathrm{c}}$, transmission coefficient $t_{l}$ approaches the eigenfunction of $H_{\mathrm{c}}$; otherwise, if it is not a resonant scattering process, all transmission coefficients $t_{l}$ vanish, and the reflection coefficient $r$ becomes -1 in accordance with Eq. (6). Therefore, we established the relationship between the scattering coefficients and the eigenfunction of the scattering center.

This method is particularly suitable for detecting edge states in topological systems. The in-gap eigenstate exponentially localizes on the boundary, and the wave function in the first unit cell is the largest. If the input channel is connected to the site at which the wave function is the largest, then the transmission probability of the first output channel will be much larger than the other output channels, and the reflection probability will be the lowest according to Eq. (6). The reflection should be as small as possible to observe large transmissions. In addition, this helps distinguish nonresonant cases, which have total reflection and zero transmission. In the following, we used 1D topological models as examples to demonstrate edge state detection.

\section{DYNAMIC DETECTION OF THE EDGE STATE}

The previous section demonstrated that the eigenfunction can be obtained by using transmission probabilities with eigenenergy known. In this section, we consider an SSH chain as a scattering center to demonstrate the proposed method. The SSH model is a prototypical 1D topological model with typical edge state features and robustness to disorder. In addition, the SSH model is also the core of numerous other topological models, including the SSH ladder [52-54], the second-order topological insulator [55-57], and the SSHHubbard model $[58,59]$. Therefore, we used the SSH model as an example without loss of generality. The Hamiltonian $H_{\mathrm{c}}$ is

$H_{\mathrm{c}}=\sum_{m=1}^{N / 2} v|2 m-1\rangle_{\mathrm{c}}\left\langle\left. 2 m\right|_{\mathrm{c}}+\sum_{m=1}^{N / 2-1} w \mid 2 m\right\rangle_{\mathrm{c}}\left\langle 2 m+\left.1\right|_{\mathrm{c}}+\right.$ H.c.

and Fig. 2 presents the scattering system. We only focused on cases in which $\mu=0$ and $k=\pi / 2$. At large $N$ limits, the system occupies a topological phase when $v<w$, which has an in-gap zero-energy edge state in the form of

$$
\left|\phi_{0}\right\rangle=\left(1-q^{2}\right)^{1 / 2} \sum_{j=1}^{N / 2}(-q)^{j-1}|2 j-1\rangle_{\mathrm{c}}
$$

$(q=v / w)$ and a trivial phase when $v>w$, for which the edge state is absent. In Appendix A, we used another scattering system to demonstrate the zero mode existing in a topological phase and vanishing in a trivial phase. In the next, we will show that the wave function of edge states can be detected using the amplitudes of outgoing waves in a topological phase, and the amplitudes are zeros in a trivial phase.

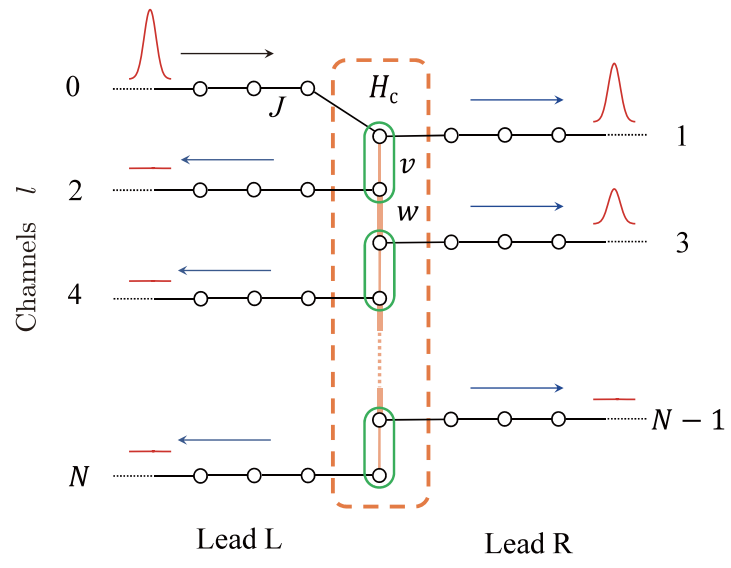

FIG. 2. A SSH model scattering center (dashed orange box) with intracell hopping $v$ (thin lines) and intercell hopping $w$ (thick lines). Even (odd) channels are placed on the left (right). In the left channels, lead site $j \in[-\infty,-1]$, and in the channel on the right lead site $j \in[1, \infty]$. The transmission probability in each lead is a geometric sequence for topological states $(v<w)$ and to be zero for trivial states $(v>w)$.

For numerical simulation, the initial state was used as the Gaussian wave packet, given by

$$
|\varphi(0)\rangle=\Omega_{0}^{-1 / 2} \sum_{j} e^{-\left(j-N_{\mathrm{c}}\right)^{2} / 2 \sigma^{2}} e^{i k_{\mathrm{c}} j}|j\rangle_{0},
$$

where $\Omega_{0}=\sum_{j} e^{-\left(j-N_{\mathrm{c}}\right)^{2} / \sigma^{2}}$ is the normalization factor, $k_{\mathrm{c}}$ is the central wave vector of the Gaussian wave packet, and the full width at half maximum of the intensity of the Gaussian wave packet is $2 \sqrt{\ln 2} \sigma$. For the simulation of plane waves, the width of the incoming wave packet must be large; otherwise, the dynamics are mixed with the dynamics near $k_{c}$. Initially, the Gaussian wave packet was centered at site $N_{\mathrm{c}}$, within the input lead. The evolved state is computed as follows,

$$
|\varphi(t)\rangle=e^{-i H t}|\varphi(0)\rangle,
$$

which yields the probability summations in each channel:

$$
p_{l}= \begin{cases}\sum_{j=1}\left|\langle\varphi(T) \mid+j\rangle_{l}\right|^{2}, & \text { odd } l \\ \sum_{j=1}\left|\langle\varphi(T) \mid-j\rangle_{l}\right|^{2}, & \text { even } l\end{cases}
$$

( $l \in[1, N])$, where $T$ is the time at which the scattering process finishes. Relations $p_{0}=|r|^{2}$ and $p_{l}=\left|t_{l}\right|^{2}(l>0)$ should be observed. Because the entire scattering system is Hermitian, it satisfies the conservation of probability. Therefore, for the input Gaussian wave packet,

$$
p_{l}= \begin{cases}{\left[1-q^{2} /\left(2-q^{2}\right)\right]^{2} q^{l-1},} & \text { odd } l \\ {\left[q^{2} /\left(2-q^{2}\right)\right]^{2},} & l=0 \\ 0, & \text { even } l \neq 0\end{cases}
$$

should be observed for $v<w$, whereas

$$
p_{l}= \begin{cases}1, & l=0 \\ 0, & l>0\end{cases}
$$



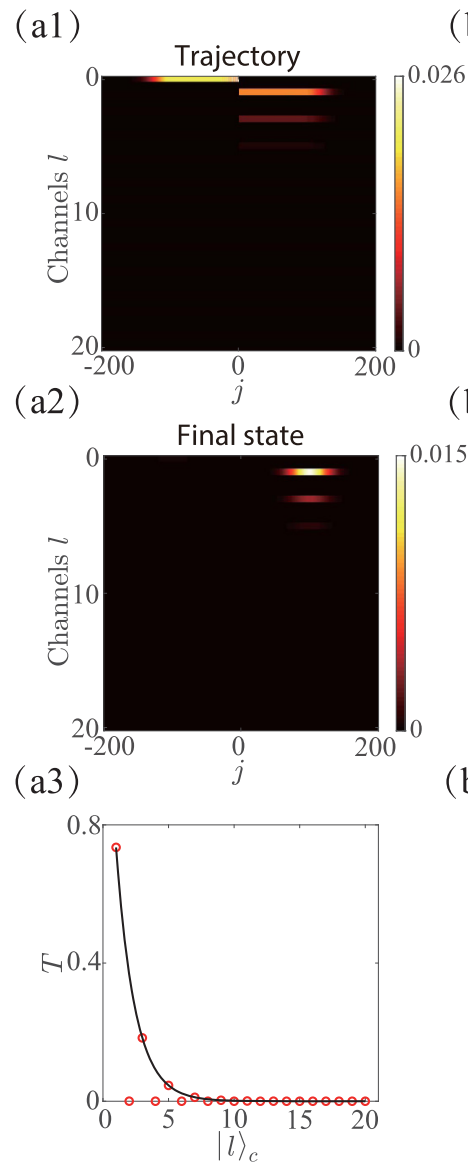

(b1)

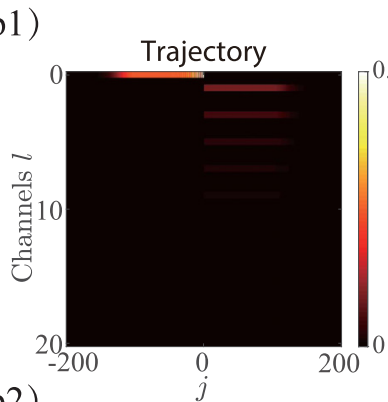

(b2)

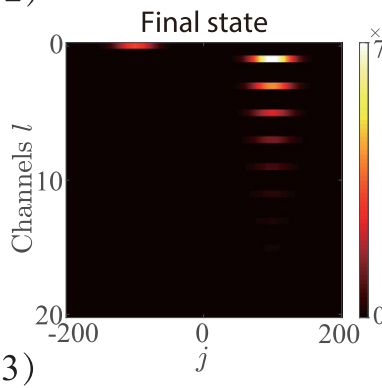

(b3)

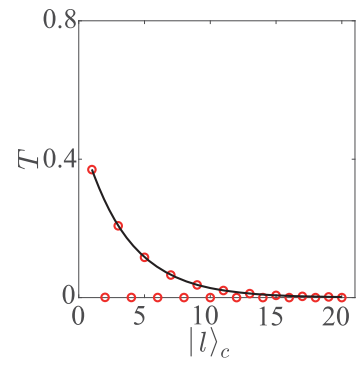

(c1)

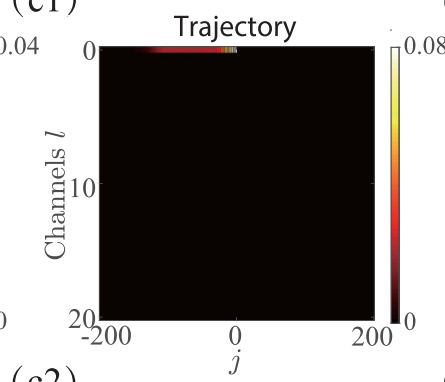

(c2)
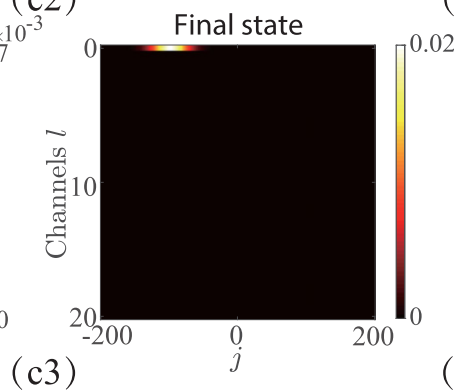

(d2)
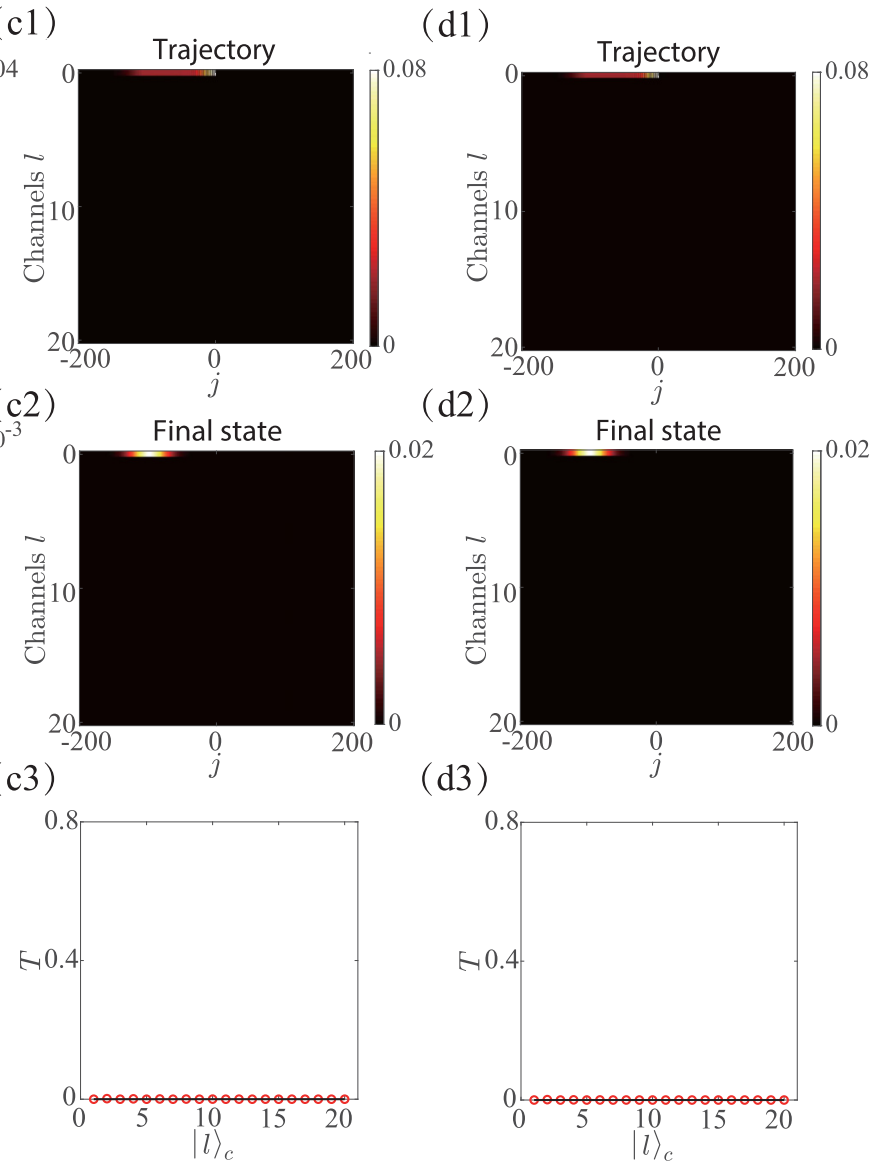

(d3)

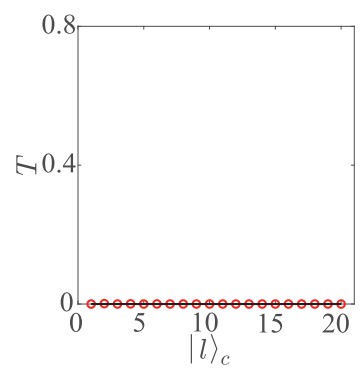

FIG. 3. Profile of trajectory and final state for the injected Gaussian wave packet with several typical sets of scattering center parameters. The system consists of a 40-site scattering center (20 unit cells) and 41200 -site tight-bounding chains. The plots present only the first 20 channels, and the transmission probabilities of the last 20 channels are almost zero. A Gaussian wave packet is initially centered at site $N_{\mathrm{c}}=-100$. The wave vector for the Gaussian wave packet is $k_{\mathrm{c}}=\pi / 2, \sigma=20$, and $\mu=0$ to ensure $E_{k}=0$ and resonance with the in-gap eigenmode. We set coupling strength $J=-0.1$. (a-d) $w=4$ and $v=2,3,5$, and 6 , respectively. (1) and (2) correspond to the trajectory and final state for each parameter. The overall structure is similar to that in Fig. 2. The vertical axis represents the channel number, and the horizontal axis represents the left and right leads with 200 sites. (3) is a comparison of transmission probabilities (red empty circle) and the eigenfunction (black line for odd sites), and the eigenfunction has been renormalized using Eq. (14). The transmission probability is a geometric series for the topological case and almost zero for the trivial case.

should be observed for $v>w$. Appendix B presents the derivation of the analytical results. To demonstrate our prediction, we plotted the numerical results for several typical sets of parameters in Fig. 3. A comparison between the analytical and numerical results revealed that the wave packet can be used to demonstrate the conclusion on the plane wave. Our conclusion is invalid near topological phase transition point $v=w$ because the zero mode is absent in small finite $\mathrm{SSH}$ chains. The outputs from the left (right) channel in the numerical simulations yield the left (right) edge state.

\section{DYNAMIC DETECTION OF THE PHASE REGION}

This section provides a scheme to experimentally detect edge states through a system of waveguide arrays. Recently, numerous studies have experimentally demonstrated $\mathrm{SSH}$ models in photonic systems [5,60]. It is based on the analogy between light propagating through a photonic crystal and a tight-binding Hamiltonian. Topological effects in some electronic systems can be observed in their photonic coun- terparts $[13,61,62]$. On a photonic platform, a single-particle state can be amplified by a large population of photons. This enables a high degree of control over the system parameters.

In the following we present a scheme to experimentally demonstrate $p_{l}$ for $H_{\mathrm{c}}$ through a 2D array made of $(N+1) \times$ $M$ equal straight waveguides, which are assumed to be weakly coupled. The waveguides can be fabricated through the direct laser writing method, and numerous 2D topological lattice systems have been implemented using this technique [63-65]. Figure 4 presents the geometry of the scattering system, which is uniform in the direction of light propagation $z$. The input and each output lead are separated to prevent the quantum tunneling effect. According to coupled-mode theory [66-68], light propagation can be described by Schrödinger-like equations, which are typically used to mimic the dynamics of a tight-binding system.

For a coupled-waveguide array with length $L$, the corresponding equations are

$$
i \frac{\partial u(z)}{\partial z}=H u(z),
$$




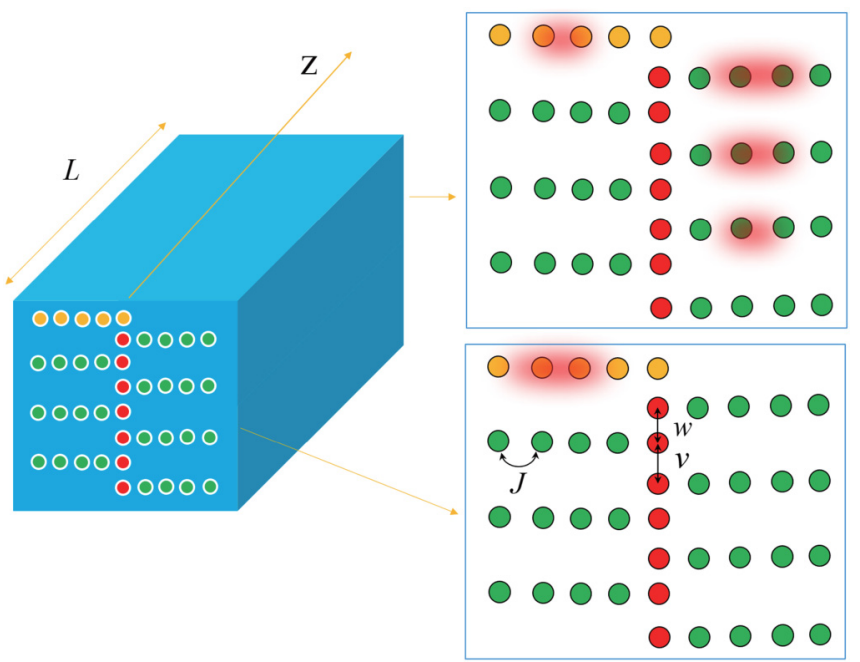

FIG. 4. Illustration of waveguide arrays for experimentally detecting the edge state. The yellow dot represents the incident chain, the green dot represents the transmission chain, and the red dot represents the SSH chain of the scattering center. Each dot represents a waveguide of length $L$, which should be designed to finish the scattering process in accordance with Eqs. (16) and (17). Coupling strength $J$ in the leads should be much smaller than coupling $v$ and $w$ in the scattering center. The bottom right and top right represent the initial and final states, respectively.

for $z \in[0, L]$, with $u(z)=\left[u_{l, i}(z), u_{\mathrm{c}, j}(z)\right]^{T}$ representing the vector, $u_{0, i}(z)(i \in[1, N])$ denoting the mode amplitude in the input waveguide, $u_{l, i}(z)(l \in[1, M], i \in[1, N])$ denoting the mode amplitude in the output waveguide, and $u_{\mathrm{c}, j}(z)(j \in$ $[1, M])$ denoting the mode amplitude in the center waveguide. Accordingly, for a given initial wave function $u(0)$,

$$
u(L)=e^{-i H L} u(0) .
$$

If $u(0)$ is used as a Gaussian function, as defined in Eq. (11), and light propagation distance $z$ is used as time $t$, then $u(L)$ corresponds to the numerical results in Fig. 3. This demonstrates that detecting edge states is possible in arrays of coupled waveguides.

We investigated the visibility and reflection to distinguish the topologically nontrivial and trivial phases. According to the theoretical analysis, the light intensity distribution in the output waveguide array (see Fig. 3) is $q$ dependent, thus obeying Eq. (14). The value of $q$ determines the visibility of two odd neighbor waveguides, defined as

$$
\mathcal{V}(\eta)=\left|\frac{\sum_{i}\left|u_{2 \eta+1, i}(L)\right|^{2}-\sum_{i}\left|u_{2 \eta-1, i}(L)\right|^{2}}{\sum_{i}\left|u_{2 \eta+1, i}(L)\right|^{2}+\sum_{i}\left|u_{2 \eta-1, i}(L)\right|^{2}}\right|,
$$

where $\eta \in[1, M / 2-1]$. The visibility in the region $q<1$ obeys

$$
\mathcal{V}(\eta)=\left(1-q^{2}\right) /\left(1+q^{2}\right)
$$

Visibility $\mathcal{V}(\eta)$ in region of $q>1$ is not well defined because all scattering chains are off-resonant with the energy levels of the scattering center and then all transmission approach zero.

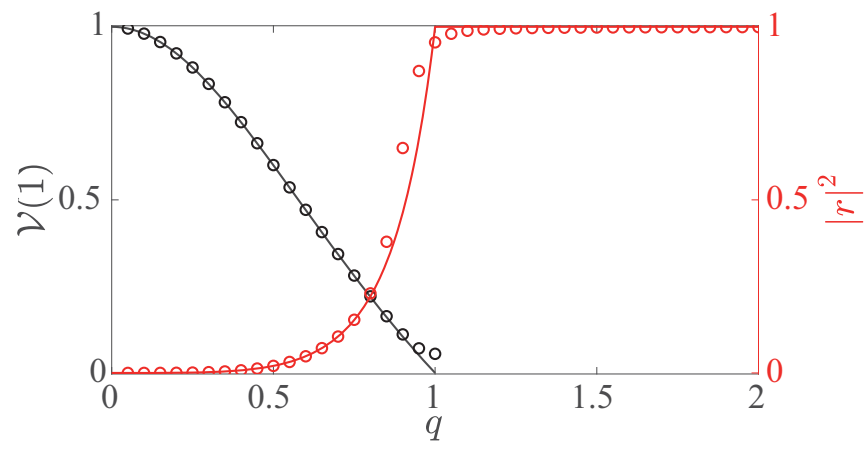

FIG. 5. $\mathcal{V}(1)$ (black) and $|r|^{2}$ (red) as functions of $q$. The lines were obtained from the analytical results of Eqs. (19) and (20), and the empty circles were obtained from the numerical simulations for an incident wave packet. A nonanalytical point was observed at $q=1$, which is the topological phase transition point, and our theory adequately explained all $q$ values except those near the phase transition point.

Reflection $|r|^{2}$ obeys

$$
|r|^{2}= \begin{cases}q^{4} /\left(2-q^{2}\right)^{2}, & q<1 \\ 1, & q>1\end{cases}
$$

which can be obtained from the analytical results. In Fig. 5 we plotted $\mathcal{V}(1)$ and $|r|^{2}$ as functions of $q$, and the results indicate that there is a nonanalytical point at $q=1$. It seems that the observation of the reflection can be the witness of the phase transition. However, the zero mode vanished near $q=1$, and our theory was not applicable. In addition, by measuring the visibility in the output waveguide array and the light intensity in the input waveguide array, the corresponding $q$ of the topological region can be measured in the experiment. To verify our prediction, we performed numerical simulation and computed $\mathcal{V}(1)$ and $|r|^{2}$ as a function of $q$, for an incident wave packet. Figure 5 presents the numerical results with empty circles, in comparison with the analytical solutions (solid line). As expected, the values of $\mathcal{V}(1)$ and $|r|^{2}$ deviated from the analytical results as $q$ approached 1 . In other regions, the theoretical results perfectly fit the numerical simulation results.

\section{NON-HERMITIAN SSH CHAIN}

In this section, we consider a non-Hermitian SSH chain as a scattering center. The non-Hermiticity arose from oppositesite imaginary potential $\pm i \gamma$. The scattering system is similar to that in Fig. 2, with only the staggered gain and loss presented as the on-site term of the scattering center. The Hamiltonian of the non-Hermitian scattering center is

$$
\mathcal{H}_{\mathrm{c}}=H_{\mathrm{c}}+i \gamma \sum_{m=1}^{N}(-1)^{m}|m\rangle_{\mathrm{c}}\left\langle\left. m\right|_{\mathrm{c}}\right.
$$

where $H_{\mathrm{c}}$ is the Hamiltonian of the Hermitian SSH model in Eq. (9). For this case, we used a strong dimerization limit, namely, $v \gg w$, and it has been shown in Ref. [69] that the 

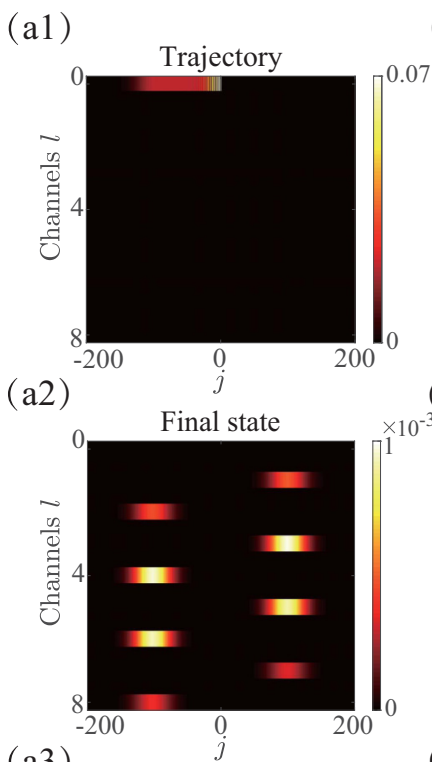

(a3)

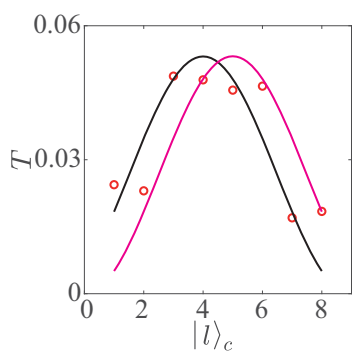

(b1)

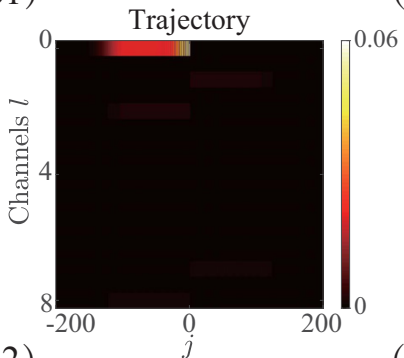

$(\mathrm{b} 2)$

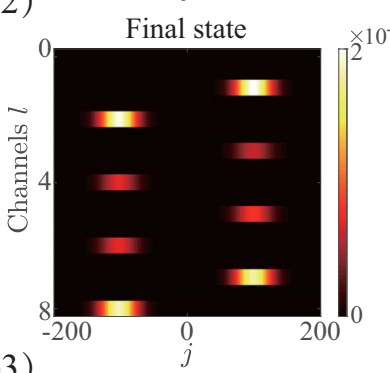

(b3)

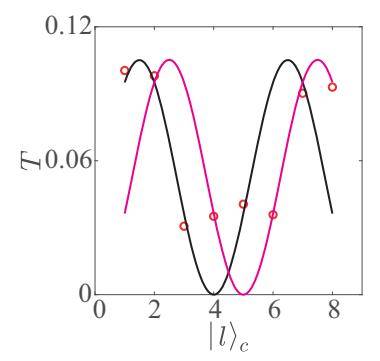

(c1)

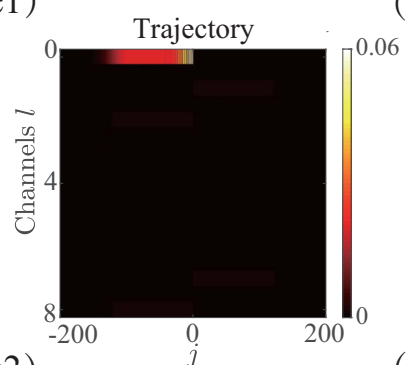

$(\mathrm{c} 2)$

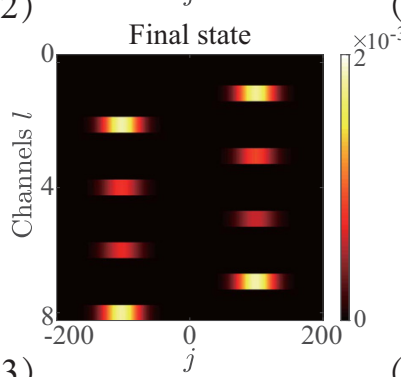

(c3)

(d3)

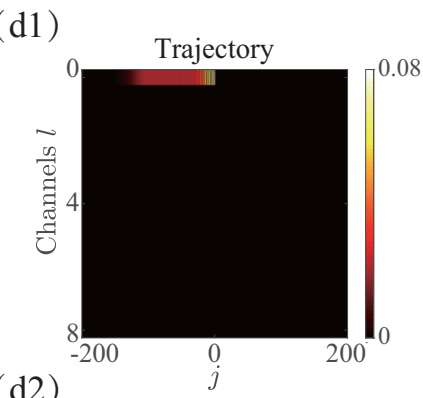

(d2)
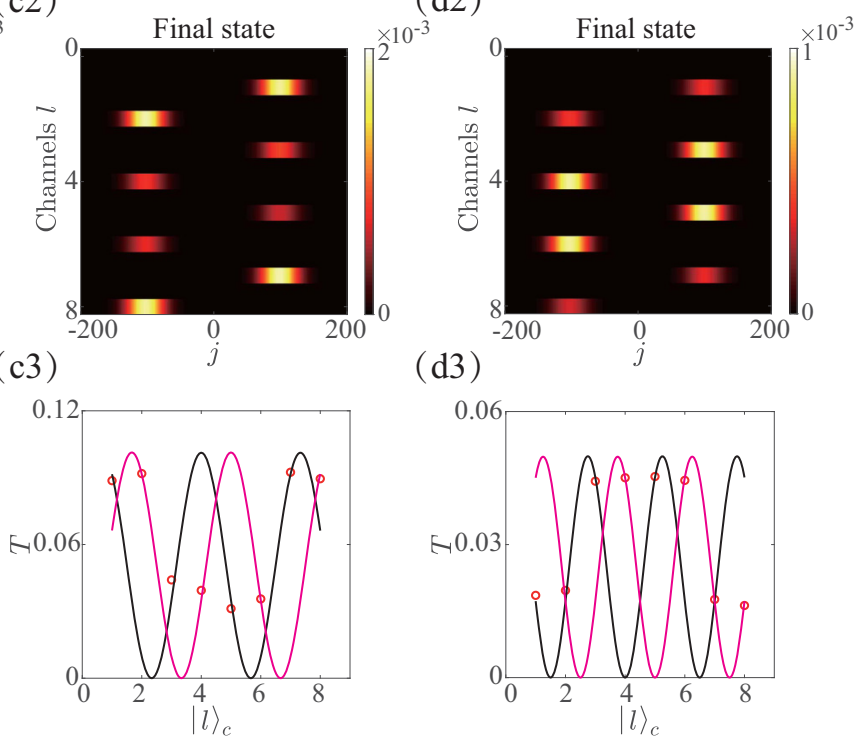

FIG. 6. Profile of trajectory and final state for the injected Gaussian wave packet with several resonance energies. A Gaussian wave packet was initially centered at the site $N_{\mathrm{c}}=-100$ and $\sigma=20$. The parameters of the scattering center were $v=40, w=2$, and $\gamma=10$. The system consists of an eight-site scattering center and 9 200-site tight-bounding chains. We set coupling strength $J=-0.1$. (a-d) $n=1,2,3$, and 4, respectively. (1) and (2) correspond to the trajectory and final state for each eigenenergy, respectively. (3) is a comparison of the transmission probabilities (red empty circle) and the eigenfunction (black line for odd sites and magenta for even sites), and the eigenfunction has been renormalized to fit the transmission probabilities. The transmission probability is a sinusoidal function with different periods, determined by energy level $n$.

eigenstates are approximately given by

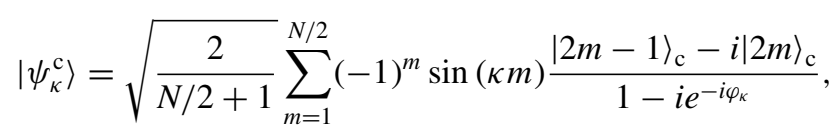

with eigenenergy given by

$$
\varepsilon_{\kappa}=\sqrt{(v-w \cos \kappa)^{2}-\gamma^{2}},
$$

where $\tan \varphi_{\kappa}=\gamma / \varepsilon_{\kappa}$ and $\kappa=(n+1) \pi /(N / 2+1), n \in$ $[0, N / 2-1]$. In Appendix A, we verified the corresponding eigenenergy. We only considered cases with real values for $\varepsilon_{\kappa}$. Spectrum $\varepsilon_{\kappa}$ was nondegenerate. The conclusion we obtained in the previous section was applicable for finite $N$ in the limit of zero $J$. For an incident plane wave with wave vector $k_{\mathrm{c}}=\pi / 2$, chemical potential $\mu$ can be adjusted to $\varepsilon_{\kappa}$. The transmission coefficients in each lead are a sinusoidal function given by

$$
\left|t_{2 m-1}\right|^{2}=\left|t_{2 m}\right|^{2} \propto \sin ^{2}(\kappa m), m \in[1, N / 2] .
$$

Different resonant $\mu$ values result in different distribution of $\left|t_{l}\right|^{2}$, which is determined by $n$.
To test this prediction, numerical simulations were performed in accordance with the procedure in the previous section. The numerical results for several typical sets of parameters are plotted in Fig. 6. A comparison between the analytical and numerical results revealed that our scattering formalism for detecting eigenfunctions was applicable to nonHermitian topological models with real-value edge states.

\section{DISCUSSION AND SUMMARY}

The prototypical SSH model was employed to demonstrate the proposed method of edge state detection. If Hamiltonian $H_{\mathrm{c}}$ is a high-order degenerate topological system with degenerate edge states at the boundary of the lattice, the resonant transmission in the scattering dynamics will yield the linear superposition of the edge states for the high-order degenerate edge states (more than one pair of degenerate left or right edge states). The proposed method can still be used to identify nontrivial topology, where transmissions in the leads exponentially decay in the topologically nontrivial phase and approach zero for all leads in the trivial phase. The method can also be applied to other topological models, including the Rice-Mele model, the Aubry-André-Harper model, and 
their generalizations, and the edge states can be detected using resonant transmission.

We developed a method to detect eigenstates in the band gap by using multichannel scattering. The connection between the eigenstate of the scattering center and the transmission and reflection amplitudes of the resonant scattering process was established at the isolated energy level. The method is applicable to both Hermitian and non-Hermitian scattering centers if the isolated energy level is real and can be used to detect the eigenstates of a system through resonant scattering. We demonstrated that the edge state in an SSH chain can be detected using photon probability in the output leads. We also proposed a scheme to demonstrate these results through a system of waveguide arrays.

\section{ACKNOWLEDGMENTS}

This work was supported by National Natural Science Foundation of China (Grants No. 11975128 and No. 11874225).

\section{APPENDIX A: DETERMINATION OF EIGENENERGY}

This Appendix demonstrates the detection of the eigenenergy of a cluster through the measurement of resonant transmission. We designed another scattering system with two leads shown in Fig. 7(a). The Hamiltonian of the output lead in system $H$ reduces to

$$
H_{\mathrm{out}}=-J \sum_{j=1}^{\infty}(|j\rangle\langle j+1|+\text { H.c. })+\mu \sum_{|j|=1}^{\infty}|j\rangle\langle j| .
$$

The joint Hamiltonian is represented by

$$
H_{\text {jnt }}=-J(|-1\rangle\langle\alpha|+| 1\rangle\langle\alpha|)+\text { H.c. },
$$

where $\alpha$ represents an arbitrary site in cluster $H_{\mathrm{c}}$ connected to the left and right leads. $N$-site scattering center $H_{\mathrm{c}}$ is defined as

$$
H_{\mathrm{c}}=\sum_{n=1}^{N} \varepsilon_{n}\left|\phi_{n}\right\rangle\left\langle\phi_{n}\right| .
$$

$H_{\mathrm{c}}$ is expressed by the eigenenergy representation. $\left|\phi_{n}\right\rangle$ denotes the eigenstate of $H_{\mathrm{c}}$ with energy $\varepsilon_{n}$ [if $H_{\mathrm{c}}$ is nonHermitian, $\left\langle\phi_{n}\right|$ in Eq. (A2) should be replaced by $\left\langle\varphi_{n}\right|$, where $\left|\varphi_{n}\right\rangle$ denotes the eigenstate of $H_{c}^{\dagger}$ with energy $\left.\varepsilon_{n}^{*}\right]$. We considered a case in which $H_{\mathrm{c}}$ has an isolated energy level at $\varepsilon_{q}$, satisfying

$$
\varepsilon_{q}=-2 J \cos k+\mu .
$$

State $\left|\psi_{k}\right\rangle$, calculated using

$$
\left|\psi_{k}\right\rangle= \begin{cases}e^{i k j}|j\rangle, & |j| \geqslant 1 \\ c\left\langle m \mid \phi_{q}\right\rangle|m\rangle, & m \in[1, N]\end{cases}
$$

is an eigenstate of $H$ with energy $\varepsilon_{q}$. Here $\{|m\rangle\}$ denotes the set of indices for the sites of the scattering center, and $c$ is a complex number, calculated using $\left\langle\alpha \mid \phi_{q}\right\rangle=c^{-1}$, which implies that $\left\langle\alpha \mid \phi_{q}\right\rangle$ must be nonzero. We observed that a perfect transition occurs without reflection under resonance, which satisfies Eq. (A3). Under nonresonance, a reflected (a)

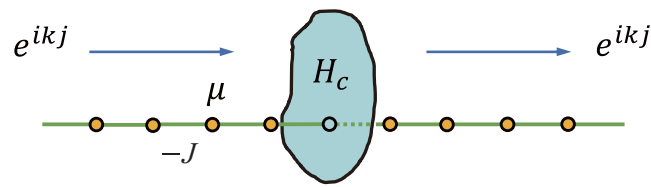

(b)

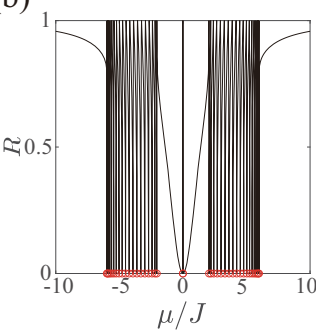

(d)

(c)

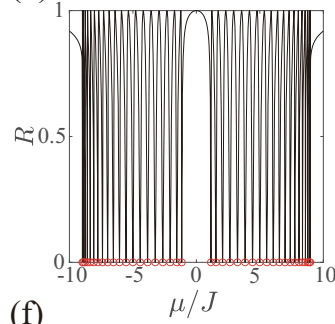

(e)
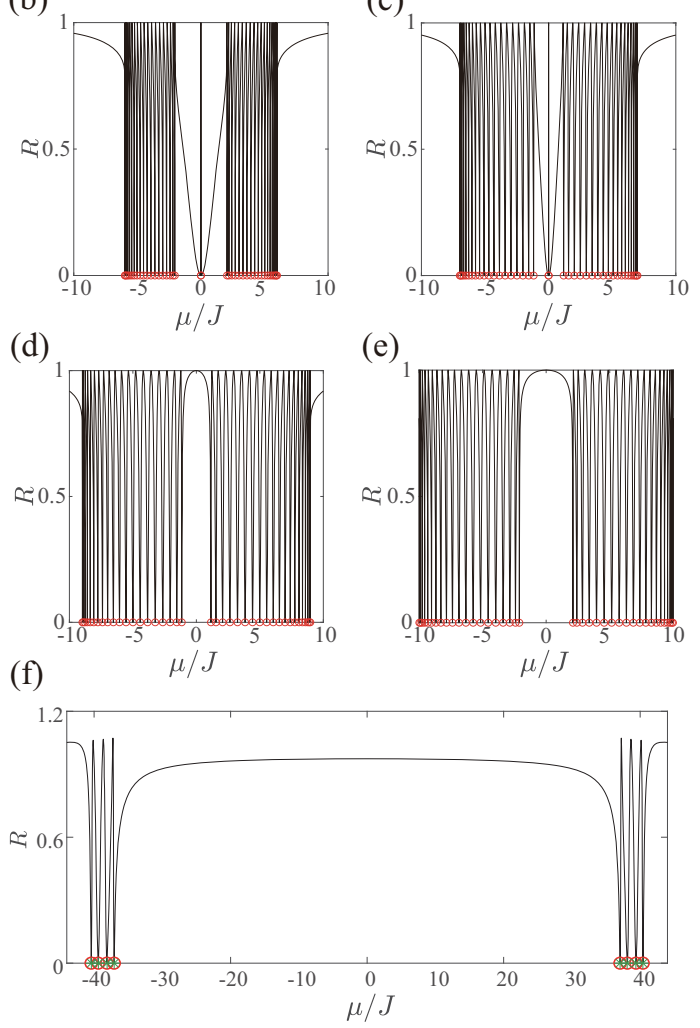

FIG. 7. Schematic of the system for determining the eigenenergy of a cluster and its corresponding numerical results. (a) Schematic of the scattering system with two leads. (b)-(e) Numerical results of the Hermitian SSH model. The parameters for the scattering center were the same as those in Fig. 3. (f) Numerical results of the non-Hermitian SSH model. The parameters for the scattering center were the same as those in Fig. 6. For all numerical results, coupling strength $J=1$, and wave vector $k=\pi / 2$. The black lines are reflections with different $\mu$ values, and the red empty circles are the corresponding eigenenergy of the scattering center. The green asterisk in panel (f) represents the analytic eigenenergy in Eq. (23).

wave appears after scattering. This result can be used to detect the eigenenergy for $H_{\mathrm{c}}$ by switching off all leads of a multichannel scattered system (Fig. 1) except 0 and 1, which is essentially an infinite chain sharing a single site with the scattering center. Because perfect transmission $(r=0)$ corresponds to on-resonance, resonant energy can be determined by scanning $\mu$ and measuring the reflection. The scattering center can be Hermitian or non-Hermitian, but the corresponding eigenenergy must be real. The coupling strength $J$ of the two leads is not limited; this differs from the requirement that the coupling strength be sufficiently low for the measurement of the eigenfunction through multichannel scattering.

To determine the validity of the method of detecting eigenenergy, we used an SSH chain and non-Hermitian SSH 
chain as scattering centers to measure the reflection of different $\mu$ values. The numerical results for several typical sets of parameters are plotted in Figs. 7(b)-7(f). A comparison between the exact eigenenergy and the numerical results indicated that all the $\mu$ values with zero reflection corresponded to eigenenergy, which suggested that our scattering method for detecting eigenenergy was applicable to both Hermitian and non-Hermitian clusters with real values. For the nonHermitian SSH model, the eigenenergy obtained by numerical calculation corresponds to the analytic expression Eq. (23). Therefore, we can experimentally detect the corresponding eigenenergy by determining whether the reflection is zero in the proposed system in Fig. 7(a).

\section{APPENDIX B: ANALYTICAL SOLUTIONS OF THE TRANSMISSION REFLECTION IN THE SSH MODEL}

The transmission coefficients of each channel are the nonnormalized eigenfunctions of the edge state. The transmission coefficients of two adjacent odd transmission channels are $-q$ times different and all transmission coefficients of even transmission channels are zero:

$$
t_{l}=\left\{\begin{array}{cc}
t_{1}(-q)^{(j-1) / 2}, & \text { odd } \\
0 . & \text { even }
\end{array} .\right.
$$

We supposed that the number of unit cells in the scattering center was sufficiently large. This would guarantee that the eigenenergy of the in-gap eigenmode would be zero, thus satisfying the resonance condition, and that the transmission coefficients would converge, enabling the use of the infinite series summation formula:

$$
\sum_{l=1}^{N}\left|t_{l}\right|^{2}=\left|t_{1}\right|^{2}\left(1+q^{2}+q^{4}+\cdots\right)=\frac{\left|t_{1}\right|^{2}}{1-q^{2}} .
$$

Because the Hamiltonian of the entire scattering system is Hermitian, the unitary scattering and probability current is conserved:

$$
|r|^{2}+\sum_{l=1}^{N}\left|t_{l}\right|^{2}=1
$$

The continuity condition Eqs. (6) and (B2) can be introduced into Eq. (B3), and we can solve that

$$
t_{1}=\frac{2\left(1-q^{2}\right)}{2-q^{2}}, \quad r=-\frac{q^{2}}{2-q^{2}} .
$$

In the numerical simulations, the probability summations in each channel $p_{l}$ reflect reflection $|r|^{2}$ and transmission $\left|t_{l}\right|^{2}$. Therefore, analytical solutions for transmission reflection can be obtained, as shown in Eq. (14).
[1] B. I. Halperin, Quantized Hall conductance, current-carrying edge states, and the existence of extended states in a twodimensional disordered potential, Phys. Rev. B 25, 2185 (1982).

[2] N. R. Cooper, J. Dalibard, and I. B. Spielman, Topological bands for ultracold atoms, Rev. Mod. Phys. 91, 015005 (2019).

[3] A. Bansil, H. Lin, and T. Das, Colloquium: Topological insulators, Rev. Mod. Phys. 88, 021004 (2016).

[4] J. K. Asbóth, L. Oroszlány, and A. Pályi, A Short Course on Topological Insulators: Band-Structure Topology and Edge States in One and Two Dimensions (Springer, New York, 2016).

[5] T. Ozawa, H. M. Price, A. Amo, N. Goldman, M. Hafezi, L. Lu, M. C. Rechtsman, D. Schuster, J. Simon, O. Zilberberg, and I. Carusotto, Topological photonics, Rev. Mod. Phys. 91, 015006 (2019).

[6] F. D. M. Haldane and S. Raghu, Possible Realization of Directional Optical Waveguides in Photonic Crystals with Broken Time-Reversal Symmetry, Phys. Rev. Lett. 100, 013904 (2008).

[7] S. Raghu and F. D. M. Haldane, Analogs of quantum-Halleffect edge states in photonic crystals, Phys. Rev. A 78, 033834 (2008).

[8] Z. Wang, Y. D. Chong, J. D. Joannopoulos, and M. Soljačić, Reflection-Free One-Way Edge Modes in a Gyromagnetic Photonic Crystal, Phys. Rev. Lett. 100, 013905 (2008).

[9] Z. Wang, Y. D. Chong, J. D. Joannopoulos, and M. Soljačić, Observation of unidirectional backscattering-immune topological electromagnetic states, Nature (London) 461, 772 (2009).

[10] W.-J. Chen, S.-J. Jiang, X.-D. Chen, J.-W. Dong, and C. T. Chan, Experimental realization of photonic topological insulator in a uniaxial metacrystal waveguide, Nat. Commun. 5, 5782 (2014).

[11] M. C. Rechtsman, J. M. Zeuner, Y. Plotnik, Y. Lumer, D. Podolsky, F. Dreisow, S. Nolte, M. Segev, and A. Szameit,
Photonic Floquet topological insulators, Nature (London) 496, 196 (2013).

[12] M. Hafezi, E. A. Demler, M. D. Lukin, and J. M. Taylor, Robust optical delay lines with topological protection, Nat. Phys. 7, 907 (2011).

[13] M. Hafezi, S. Mittal, J. Fan, A. Migdall, and J. M. Taylor, Imaging topological edge states in silicon photonics, Nat. Photonics 7, 1001 (2013).

[14] K. Fang, Z. Yu, and S. Fan, Realizing effective magnetic field for photons by controlling the phase of dynamic modulation, Nat. Photonics 6, 782 (2012).

[15] J. Koch, A. A. Houck, K. L. Hur, and S. M. Girvin, Timereversal-symmetry breaking in circuit-QED-based photon lattices, Phys. Rev. A 82, 043811 (2010).

[16] A. Petrescu, A. A. Houck, and K. Le Hur, Anomalous Hall effects of light and chiral edge modes on the Kagomé lattice, Phys. Rev. A 86, 053804 (2012).

[17] A. B. Khanikaev, S. H. Mousavi, W.-K. Tse, M. Kargarian, A. H. MacDonald, and G. Shvets, Photonic topological insulators, Nat. Mater. 12, 233 (2013).

[18] M. Sato, Topological properties of spin-triplet superconductors and Fermi surface topology in the normal state, Phys. Rev. B 79, 214526 (2009).

[19] D. Pesin and L. Balents, Mott physics and band topology in materials with strong spin-orbit interaction, Nat. Phys. 6, 376 (2010).

[20] X. Wan, A. M. Turner, A. Vishwanath, and S. Y. Savrasov, Topological semimetal and Fermi-arc surface states in the electronic structure of pyrochlore iridates, Phys. Rev. B 83, 205101 (2011).

[21] L. A. Wray, S.-Y. Xu, Y. Xia, D. Hsieh, A. V. Fedorov, Y. S. Hor, R. J. Cava, A. Bansil, H. Lin, and M. Z. Hasan, 
A topological insulator surface under strong coulomb, magnetic and disorder perturbations, Nat. Phys. 7, 32 (2011).

[22] S.-Y. Xu, Y. Xia, L. A. Wray, S. Jia, F. Meier, J. H. Dil, J. Osterwalder, B. Slomski, A. Bansil, H. Lin, R. J. Cava, M. Z. Hasan, Topological phase transition and texture inversion in a tunable topological insulator, Science 332, 560 (2011).

[23] L. Wu, M. Brahlek, R. V. Aguilar, A. V. Stier, C. M. Morris, Y. Lubashevsky, L. S. Bilbro, N. Bansal, S. Oh, and N. P. Armitage, A sudden collapse in the transport lifetime across the topological phase transition in $\left(\mathrm{Bi}_{1-x} \mathrm{In}_{x}\right)_{2} \mathrm{Se}_{3}$, Nat. Phys. 9, 410 (2013).

[24] T. Kitagawa, E. Berg, M. Rudner, and E. Demler, Topological characterization of periodically driven quantum systems, Phys. Rev. B 82, 235114 (2010).

[25] Z. Gu, H. A. Fertig, D. P. Arovas, and A. Auerbach, Floquet Spectrum and Transport through an Irradiated Graphene Ribbon, Phys. Rev. Lett. 107, 216601 (2011).

[26] N. H. Lindner, G. Refael, and V. Galitski, Floquet topological insulator in semiconductor quantum wells, Nat. Phys. 7, 490 (2011).

[27] B. Dóra, J. Cayssol, F. Simon, and R. Moessner, Optically Engineering the Topological Properties of a Spin Hall Insulator, Phys. Rev. Lett. 108, 056602 (2012).

[28] Y. T. Katan, and D. Podolsky, Modulated Floquet Topological Insulators, Phys. Rev. Lett. 110, 016802 (2013).

[29] A. Kundu and B. Seradjeh, Transport Signatures of Floquet Majorana Fermions in Driven Topological Superconductors, Phys. Rev. Lett. 111, 136402 (2013).

[30] Y. H. Wang, H. Steinberg, P. Jarillo-Herrero, and N. Gedik, Observation of Floquet-Bloch states on the surface of a topological insulator, Science 342, 453 (2013).

[31] P. M. Perez-Piskunow, G. Usaj, C. A. Balseiro, and L. E. F. F. Torres, Floquet chiral edge states in graphene, Phys. Rev. B 89, 121401(R) (2014).

[32] R. Wang, B. Wang, R. Shen, L. Sheng, and D. Y. Xing, Floquet Weyl semimetal induced by off-resonant light, Europhys. Lett. 105, 17004 (2014).

[33] B. Bernevig and T. Hughes, Topological Insulators and Topological Superconductors (Princeton University, Princeton, NJ, 2013).

[34] T. Ozawa, and I. Carusotto, Anomalous and Quantum Hall Effects in Lossy Photonic Lattices, Phys. Rev. Lett. 112, 133902 (2014).

[35] M. Hafezi, Measuring Topological Invariants in Photonic Systems, Phys. Rev. Lett. 112, 210405 (2014).

[36] W. Hu, J. C. Pillay, K. Wu, M. Pasek, P. P. Shum, and Y. D. Chong, Measurement of a Topological Edge Invariant in a Microwave Network, Phys. Rev. X 5, 011012 (2015).

[37] M. Atala, M. Aidelsburger, J. T. Barreiro, D. Abanin, T. Kitagawa, E. Demler, and I. Bloch, Direct measurement of the Zak phase in topological Bloch bands, Nat. Phys. 9, 795 (2014).

[38] L. Duca, T. Li, M. Reitter, I. Bloch, M. Schleier-Smith, and U. Schneider, An Aharonov-Bohm interferometer for determining Bloch band topology, Science 347, 288 (2015).

[39] M. Aidelsburger, M. Lohse, C. Schweizer, M. Atala, J. T. Barreiro, S. Nascimbène, N. R. Cooper, I. Bloch, and N. Goldman, Measuring the Chern number of Hofstadter bands with ultracold bosonic atoms, Nat. Phys. 11, 162 (2015).
[40] S. Mittal, S. Ganeshan, J. Fan, A. Vaezi, and M. Hafezi, Measurement of topological invariants in a 2D photonic system, Nat. Photonics 10, 180 (2016).

[41] F. Z.-Nejad and R. Fleury, Topological Fano Resonances, Phys. Rev. Lett. 122, 014301 (2019).

[42] M. Reisner, D. H. Jeon, C. Schindler, H. Schomerus, F. Mortessagne, U. Kuhl and T. Kottos, Self-Shielded Topological Receiver Protectors, Phys. Rev. Appl. 13, 034067 (2020).

[43] D. Meidan, T. Micklitz, and P. W. Brouwer, Topological classification of adiabatic processes, Phys. Rev. B 84, 195410 (2011).

[44] I. C. Fulga, F. Hassler, and A. R. Akhmerov, Scattering theory of topological insulators and superconductors, Phys. Rev. B 85, 165409 (2012).

[45] M. S. Rudner, N. H. Lindner, E. Berg, and M. Levin, Anomalous Edge States and the Bulk-Edge Correspondence for Periodically Driven Two-Dimensional Systems, Phys. Rev. X 3, 031005 (2013).

[46] M. Pasek and Y. D. Chong, Network models of photonic Floquet topological insulators, Phys. Rev. B 89, 075113 (2014).

[47] L. Jin, Topological phases and edge states in a non-Hermitian trimerized optical lattice, Phys. Rev. A 96, 032103 (2017).

[48] H. C. Wu, L. Jin, and Z. Song, Inversion symmetric nonHermitian Chern insulator, Phys. Rev. B 100, 155117 (2019).

[49] M. Xiao, Z. Q. Zhang, and C. T. Chan, Surface Impedance and Bulk Band Geometric Phases in One-Dimensional Systems, Phys. Rev. X 4, 021017 (2014).

[50] A. V. Poshakinskiy, A. N. Poddubny, and M. Hafezi, Phase spectroscopy of topological invariants in photonic crystals, Phys. Rev. A 91, 043830 (2015).

[51] J. Arkinstall, M. H. Teimourpour, L. Feng, R. El-Ganainy, and H. Schomerus, Topological tight-binding models from nontrivial square roots, Phys. Rev. B 95, 165109 (2017).

[52] K. L. Zhang, H. C. Wu, L. Jin, and Z. Song, Topological phase transition independent of system non-Hermiticity, Phys. Rev. B 100, 045141 (2019).

[53] H. C. Wu, X. M. Yang, L. Jin, and Z. Song, Untying links through anti-parity-time-symmetric coupling, Phys. Rev. B 102, 161101(R) (2020).

[54] H. C. Wu, L. Jin, and Z. Song, Topology of an anti-parity-time symmetric non-Hermitian Su-Schrieffer-Heeger model, Phys. Rev. B 103, 235110 (2021).

[55] B. Y. Xie, H. F. Wang, H. X. Wang, X. Y. Zhu, J. H. Jiang, M. H. $\mathrm{Lu}$, and Y. F. Chen, Second-order photonic topological insulator with corner states, Phys. Rev. B 98, 205147 (2018).

[56] R. W. Bomantara, L. W. Zhou, J. X. Pan, and J. B. Gong, Coupled-wire construction of static and Floquet second-order topological insulators, Phys. Rev. B 99, 045441 (2019).

[57] T. Fukui, Dirac fermion model associated with a second-order topological insulator, Phys. Rev. B 99, 165129 (2019).

[58] M. D. Liberto, A. Recati, I. Carusotto and C. Menotti, Twobody physics in the Su-Schrieffer-Heeger model, Phys. Rev. A 94, 062704 (2016).

[59] M. D. Liberto, A. Recati, I. Carusotto and C. Menotti, Twobody bound and edge states in the extended SSH Bose-Hubbard model, Eur. Phys. J. Spec. Top. 226, 2751 (2017).

[60] L. Lu, J. D. Joannopoulos, and M. Soljačić, Topological photonics, Nat. Photonics 8, 821 (2014).

[61] M. C. Rechtsman, Y. Plotnik, J. M. Zeuner, D. Song, Z. Chen, A. Szameit, and M. Segev, Topological Creation and 
Destruction of Edge States in Photonic Graphene, Phys. Rev. Lett. 111, 103901 (2013).

[62] Y. Plotnik, M. C. Rechtsman, D. Song, M. Heinrich, J. M. Zeuner, S. Nolte, Y. Lumer, N. Malkova, J. Xu, A. Szameit, Z. Chen, and M. Segev, Observation of unconventional edge states in "photonic graphene," Nat. Mater. 13, 57 (2014).

[63] J. Noh, S. Huang, D. Leykam, Y. D. Chong, K. P. Chen, and M. C. Rechtsman, Experimental observation of optical Weyl points and Fermi arc-like surface states, Nat. Phys. 13, 611 (2017).

[64] S. Stützer, Y. Plotnik, Y. Lumer, P. Titum, N. H. Lindner, M. Segev, M. C. Rechtsman, and A. Szameit, Photonic topological Anderson insulators, Nature (London) 560, 461 (2018).
[65] J. Noh, W. A. Benalcazar, S. Huang, M. J. Collins, K. P. Chen, T. L. Hughes, and M. C. Rechtsman, Topological protection of photonic mid-gap defect modes, Nat. Photonics 12, 408 (2018).

[66] H. A. Haus and W. Huang, Coupled-mode theory, Proc. IEEE 79, 1505 (1991).

[67] S. Fan, W. Suh, and J. D. Joannopoulos, Temporal coupledmode theory for the Fano resonance in optical resonators, J. Opt. Soc. Am. A 20, 569 (2003).

[68] J. D. Joannopoulos, S. G. Johnson, J. N. Winn, and R. D. Meade, Photonic Crystals: Modeling the Flow of Light (Princeton University, Princeton, NJ, 2008).

[69] K. L. Zhang, P. Wang, G. Zhang, and Z. Song, Simple harmonic oscillation in a non-Hermitian Su-Schrieffer-Heeger chain at the exceptional point, Phys. Rev. A 98, 022128 (2018). 